\title{
Pesquisas sobre Estado da Arte em CTS: Análise Comparativa com a Produção em Periódicos Nacionais
}

(Research on State of the Art in STS: Comparative Analysis with Production in National Journals)

\section{LILLIANE MIRANDA FREITAS ${ }^{1}$ e EVANDRO GHEDIN²}

${ }^{1}$ Universidade Federal do Pará (lilliane@ufpa.br)

${ }^{2}$ Universidade Estadual de Roraima (evandroghedin@gmail.com)

Resumo. Esta pesquisa teve como objetivo fazer um levantamento e síntese de pesquisas sobre estado da arte em CTS publicadas de 2008 a 2013 e analisa-las comparativamente com a produção com enfoque CTS publicada em quatro periódicos nacionais no período de 2009 a 2013. Foram sintetizados 13 trabalhos sobre estado da arte considerando: as bases de dados, período, objetivos e categorias analisadas; e analisados comparativamente com 21 artigos dos quatro periódicos a partir dos descritores: modalidade de formação e sujeitos investigados, foco temático, metodologias de coleta de dados e referenciais teóricos. Os resultados da comparação entre as pesquisas de estado da arte e as pesquisas do quinquênio considerado nos permitiram inferir que: a linha de pesquisa CTS permanece em processo de expansão; a Educação Básica e os professores continuam sendo a modalidade de formação e sujeitos mais investigados; as pesquisas têm priorizado como foco temático as implementações da abordagem CTS em sala de aula e as reflexões teóricas em busca de fundamentação baseada em autores nacionais, em especial nos pressupostos freireanos.

Abstract. This study aimed to survey and research summary of state of the art in STS published from 2008 to 2013 and analyzes them compared to production with STS focus on four national journals published in the period 2009 to 2013 were synthesized 13 jobs on state of the art considering: databases, period, goals and analyzed categories; analyzed and compared to 21 articles of the four journals from the descriptors: training mode and subjects investigated, thematic focus, data collection methodologies and theoretical frameworks. The results of the comparison between the state of the art research and five years of research considered allowed us to infer that: the STS line of research remains in the expansion process; Basic Education and the teachers remain the type of training and more research subjects; research has prioritized as thematic focus implementations of the STS approach in classroom and theoretical reflections in search of reasoning based on national authors, especially in Freire's assumptions.

Palavras-chaves: CTS, revisão de literatura, periódicos, estado da arte

Keywords: STS, literature review, periodicals, state of the art

\section{Introdução}

As discussões sobre educação em Ciência-Tecnologia-Sociedade (CTS) emergiram na segunda metade do século $\mathrm{XX}$, fruto da insatisfação e críticas de resultados ambientais e sociais negativos causados pela ciência e tecnologia (AULER; BAZZO, 2001). A partir daí cada vez mais o ensino de ciências tem sido pensado em articulação com questões que relacionam ciência, tecnologia, sociedade e ambiente e, cada vez mais cresce as produções acadêmicas sobre CTS (SANTOS; MORTIMER, 2002).

A emergência dos estudos CTS data desde a década de 1980 em âmbito internacional e em âmbito nacional desde 1990, com a publicação da primeira dissertação de mestrado brasileira defendida no campo da educação CTS em 1992, e a 
partir daí se lançou num rápido crescimento (CACHAPUZ et al., 2008; MIRANDA, 2013a).

Ao longo desses últimos 20 anos, no contexto brasileiro, constata-se que o campo CTS vem se consolidando e isto pode ser verificado pelo crescente acúmulo de produções em periódicos internacionais, nacionais, em atas de eventos acadêmicos, em teses e dissertações. O número de grupos de pesquisa sobre educação CTS registrados no CNPq também tem crescido, segundo Miranda (2013b), até o primeiro semestre de 2012 havia 66 grupos de pesquisa certificados por suas instituições de origem.

Devido essa rápida expansão do campo CTS torna-se cada vez mais necessário conhecer a produção acadêmica nacional, implicada em revisão periódica de tal produção, identificando seus pressupostos teórico-metodológicos, tendências, objetivos e temáticas de pesquisa, principais resultados e possíveis contribuições para a melhoria do ensino e da formação, bem como o desenvolvimento de novos campos de investigação. Partindo desta preocupação, diversos pesquisadores têm se debruçado sobre o volume de produções na área CTS, buscado realizar revisões do tipo estado da arte, para, com os mais diferentes objetivos, sistematizar e avaliar a produção e disseminação de pesquisas nas suas mais variadas formas de publicação.

Nestes termos, temos como questão de investigação: o que dizem as pesquisas de estado da arte sobre a produção do campo CTS e o que nos últimos cinco anos tem sido produzido na área? Diante disso, a presente pesquisa tem dois objetivos: (i) fazer um levantamento e síntese de trabalhos que tratam sobre o estado da arte da pesquisa em CTS em diferentes bases de dados, (ii) discutir comparativamente os resultados desses trabalhos com a análise da produção sobre CTS do último quinquênio (20092013) em quatro periódicos nacionais da área do Ensino de Ciências.

\section{O que dizem as pesquisas do tipo estado da arte sobre o campo CTS?}

Segundo Ferreira (2002) as pesquisas em "Estado da Arte" ou "Estado do Conhecimento" são denominadas bibliográficas, onde investigam e discutem acervos acadêmicos de determinado campo do conhecimento. Elas objetivam obter respostas sobre os aspectos e as dimensões que vêm sendo estudadas e/ou privilegiadas ao longo de períodos distintos, assim como as formas e condições em que as publicações vêm sendo produzidas. Esse tipo de método apresenta caráter inventariante descritivo da produção acadêmica e científica sobre o tema a ser investigado e consistem num 
balanço do conhecimento, baseado na análise comparativa de vários trabalhos, sobre uma determinada temática (ANDRÉ, 1999).

Realizamos um levantamento nas atas das edições de 2009, 2011 e 2013 do Encontro Nacional de Pesquisa em Educação em Ciências (ENPEC) e em cinco periódicos nacionais: Ensaio: Pesquisa em Educação em Ciências (Ensaio), a Revista Brasileira de Pesquisa em Educação em Ciências (RBPEC), Investigações em Ensino de Ciências (IENCI), Ciência \& Educação (C\&E) e Alexandria Revista de Educação em Ciência e Tecnologia, nos quais encontramos 10 trabalhos com a temática CTS. Encontramos ainda 3 trabalhos que não estavam nessas plataformas inicialmente elegidas, mas que se relacionavam às pesquisas. Assim, foram selecionados 13 trabalhos de estado da arte em CTS publicados no período de 2008 a 2013, para que fossem analisados paralelamente ao último quinquênio de produção sobre a temática.

Faremos neste espaço uma síntese das 13 pesquisas sobre estado da arte em CTS publicadas nos periódicos nacionais (6) e nas atas de eventos nacionais da área de Educação em Ciências (7), evidenciando os objetivos da revisão, em que base de dados foram realizadas as pesquisas (teses, dissertações, periódicos, anais de eventos), o período analisado e algumas das principais categorias analisadas.

O levantamento realizado por Cachapuz et al. (2008) teve como objetivo evidenciar a evolução da Pesquisa em Educação em Ciência, em particular da linha de estudos CTS, no período de uma década (1993-2002), através da análise de três revistas de circulação internacional. Foram encontradas a partir da análise de 152 artigos, 11 linhas de pesquisa, sendo as linhas dominantes a aprendizagem de conceitos, a filosofia da ciência e a linguagem. No entanto, somente a linha de estudos CTS (com 9 artigos) triplicou seu número de ocorrências na década analisada. Os autores aprofundaram a análise dos trabalhos em CTS para identificar a principal orientação da pesquisa (para prática, para teoria ou para políticas), a perspectiva CTS evidenciada e as principais conclusões, reflexões ou implicações apontadas nas investigações.

A pesquisa desenvolvida por Sutil et al. (2008) teve como objetivo discutir aspectos epistemológicos, sociológicos e educacionais dos pressupostos dos movimentos CTS e CTSA no ensino de Ciências/Física, a partir da análise de artigos publicados em 5 periódicos nacionais no período de 2000 a 2007. Os resultados destacados pelos autores a partir da análise de 58 trabalhos referem-se a: (i) concepções educacionais; (ii) objetivos educacionais relacionados aos movimentos CTS e CTSA; 
(iii) organização curricular; (iv) atividades educacionais desenvolvidas; (v) avaliação e (vi) disciplinas escolares.

A pesquisa bibliográfica desenvolvida por Auler et al. (2009) buscou aprofundar aspectos teórico-metodológicos da relação entre temas geradores, conceituados por Paulo Freire e temas articulados ao enfoque CTS, utilizando-se como critério de seleção dos artigos a necessidade dos mesmos abarcarem análises de implementações, na escola, de propostas curriculares balizadas pelo referencial freireano ou pelo enfoque CTS. Tiveram como fontes de pesquisa: anais de três eventos específicos ocorridos em 2004, 2005 e 2006 (83 artigos), coletânea de cadernos do Movimento de Reorientação Curricular - Relatos de Práticas (1992) e guias didáticos do Grupo Argo de Renovação Educativa. Os resultados foram agrupados em cinco categorias: i) abrangência dos temas; ii) surgimento dos temas; ii) disciplinas envolvidas na estruturação do tema, iv) relação tema/conteúdo e v) conteúdo tradicional designado de tema.

O trabalho de revisão realizado por Hunsche et al. (2009) analisou 27 artigos de 3 periódicos nacionais da área da Educação em Ciências no período de 1998 a 2008, com objetivo de verificar quais os encaminhamentos dados na educação em ciências com enfoque CTS no contexto brasileiro. Os resultados foram agrupados em três categorias: 1) Implementações; 2) Concepções e 3) Pressupostos/Reflexões.

Fagundes et al. (2009) tiveram como objetivo verificar que abordagens as investigações para o Ensino de Ciências na perspectiva CTS/CTSA vem tomando, em relação aos temas/assuntos abordados e para que níveis de escolaridade as Atividades Didáticas são dirigidas. Eles analisaram 108 trabalhos sobre a perspectiva CTS/CTSA apresentados em seis edições do evento ENPEC-Encontro Nacional de Pesquisa em Educação em Ciência (1997 a 2007). Os trabalhos foram agrupados e analisados em 6 categorias: concepções, currículo, livros didáticos, artigos publicados em periódicos/trabalhos de eventos, revisão bibliográfica e atividades didáticas.

O levantamento realizado por Pansera-de-Araújo et al. (2009) teve como objetivo compreender a extensão e disseminação da perspectiva CTS, e para tal analisaram as produções publicadas nas atas dos eventos brasileiros sobre Ensino de Biologia, Química, Física e Ciências, no período de 2003 a 2006. Os resultados foram analisados em relação às áreas das Ciências mais envolvidas, às instituições dos autores, referências utilizadas e abordagens dos autores referência.

Abreu et al. (2009) analisaram em 10 revistas da área de Ensino de Ciências a produção nacional em CTS em todos os números publicados nas revistas, que então 
abrangeu o período de 1979 a 2008. Os autores identificam nos 23 artigos analisados as filiações teóricas às linhas de CTS ou CTSA, distribuição temporal, natureza dos trabalhos, principais temas, sujeitos investigados, autores mais citados e referenciais teórico-metodológicos.

Dando continuidade a este último estudo, Abreu et al. (2013) incluem em suas análises além do levantamento realizado em 10 revistas de pesquisa em ensino de ciências no âmbito nacional, a análise de 4 periódicos internacionais e as atas de dois encontros nacionais da área no período de 1980 a 2008. Os autores analisaram 135 trabalhos (23 de periódicos nacionais, 57 dos internacionais, 55 dos eventos) quanto ao quantitativo de publicações entre as revistas e encontros, à distribuição temporal, natureza e objetivos das pesquisas, sujeitos investigados, autores mais citados, filiações às perspectivas CTS ou CTSA e as abordagens/conceituações de CTS mais frequentes e influentes no campo de pesquisa brasileiro.

A pesquisa de Lopes et al. (2009) buscou mapear trabalhos relacionados à temática CTS nas edições entre 2000 e 2007 dos ENPECs e os EPEFs (Encontro de Pesquisa em Ensino de Física), com objetivo de analisar a produção acadêmica e suas possíveis tendências. Categorizaram os 128 trabalhos em seis tendências: 1) Formação de professores; 2) Materiais Didáticos e Paradidáticos; 3) Ensino em Espaços não formais; 4) Ensino por temas; 4) História e Filosofia da Ciência; 5) Educação Ambiental; e, 6) Estado da Arte.

Strieder e Kawamura (2011) realizaram um levantamento de pesquisas sobre CTS apresentadas no periódico brasileiro Ciência \& Educação, no período de 2000 a 2010, com objetivo de analisar como pesquisadores em Ensino de Ciências têm abordado as relações CTS. Os 12 trabalhos analisados foram agrupados em três categorias: (i) Distanciamento do conhecimento científico-tecnológico do conjunto da sociedade; (ii) Participação Social nos rumos do desenvolvimento científicotecnológico; (iii) Papel do conhecimento científico nas decisões sobre Ciência e Tecnologia.

O estado da arte da abordagem CTS realizado por Ferst (2013) revisou artigos publicados em 3 revistas nacionais e 4 internacionais, no período de 2003 a 2012, com objetivo de verificar a inserção da abordagem CTS nos anos iniciais do Ensino Fundamental. A autora destacou nos resultados a carência de publicações que tratam da abordagem CTS nos anos iniciais do Ensino Fundamental, a crescente preocupação dos 
pesquisadores em discutir as concepções e crenças relacionadas à abordagem CTS e ainda a ausência de trabalhos interdisciplinares.

Gonçalves e Menezes (2013) apresentaram um estudo sobre perspectivas e possibilidades do enfoque CTS no ensino de ciências da escola básica a partir dos resultados de pesquisas desenvolvidas no Brasil no período de 2002 a 2012 em três periódicos nacionais. Emergiram quatro categorias como resultados dos 14 artigos analisados, relativas às propostas CTS elaboradas no contexto da educação brasileira: (i) concepções (procura compreender a natureza social da Ciência e da Tecnologia e o entendimento das relações CTS); (ii) pressupostos (busca identificar factíveis aproximações entre teorias educativas e o enfoque CTS); (iii) abordagem temática (trata de temas/projetos com relevância social trabalhados em sala de aula); e (iv) referências curriculares (evidencia as relações CTS com implementações curriculares).

A pesquisa realizada por Chrispino et al. (2013) buscou identificar os trabalhos mais citados como fontes de referência em 22 periódicos nacionais na área de Ensino de Ciências no período de 1996 a 2010. Os autores analisaram 88 artigos que forneceram um total de 394 fontes bibliográficas, das quais eles listaram os 13 trabalhos mais citados. Além disso, ressaltaram que há predominância de autores nacionais como fonte de pesquisa nos trabalhos; que não são referidos os autores fundadores na área; e que não é possível perceber nos trabalhos a interdisciplinaridade e a contextualização características da área CTS.

A partir da síntese dos trabalhos acima, verificamos que as pesquisas em estado da arte analisam produções da área CTS publicadas desde 1980 a 2013, sendo o período de produção mais analisado as décadas de 1990 e 2000, a maioria levando em conta o intervalo de uma década. Através desses estudos é possível notar que a produção sobre CTS em periódicos nacionais é bem recente, pois segundo Abreu et al. (2013), o primeiro artigo publicado na área foi em 2001. Já a produção internacional conta com cerca de três décadas de publicações, tendo nas décadas de oitenta e noventa um volume mais representativo de trabalhos do que os anos 2000 (ABREU et al., 2013).

Os trabalhos analisam os estudos CTS na área de Ensino de Ciências em geral, apenas a pesquisa de Sutil et al (2008) analisa de forma particular a abordagem CTS no ensino de Física.

Quanto às bases de dados, a maioria das pesquisas realizou levantamentos em periódicos nacionais e atas de eventos. Os periódicos nacionais mais investigados foram as revistas Ciência \& Educação (Universidade Estadual Paulista), Revista Brasileira de 
Pesquisa em Educação em Ciências (Associação Brasileira de Pesquisa em Educação em Ciências), Ensaio: Pesquisa em Educação em Ciências (Universidade Federal de Minas Gerais) e Investigações em Ensino de Ciências (Universidade Federal do Rio Grande do Sul). Podemos afirmar que esses 4 periódicos se constituem em importantes referências nacionais na área de Educação em Ciências, privilegiados pelos pesquisadores como os mais importantes e representativos veículos de publicação nacional. São periódicos disponibilizados em formato eletrônico e possuem periodicidade quadrimestral.

O evento privilegiado pelas pesquisas para análise de atas foi o Encontro Nacional de Pesquisa em Educação em Ciências (ENPEC) promovido pela Associação Brasileira de Pesquisa em Educação em Ciências. Da mesma maneira, este evento é considerado pelos pesquisadores como o mais importante evento acadêmico da área, pois além de ser classificado no $\mathrm{CNPq}$, como qualis $\mathrm{A}$, o evento reúne os pesquisadores de todas as áreas da Educação em Ciências, para discussão dos problemas, resultados de pesquisa e contribuições das mesmas para o Ensino de Ciências.

Em relação aos objetivos, verificamos na síntese das pesquisas que as revisões ocorreram pelos mais diversos objetivos, no entanto percebemos uma preocupação comum a quase todas as pesquisas que é analisar principalmente as abordagens teóricometodológicas dos trabalhos especialmente quanto às filiações teóricas das abordagens, conceituações e perspectivas CTS/CTSA e em identificar os principais referenciais da área por elas utilizadas.

Considerando o expressivo volume de análises e resultados das pesquisas do tipo estado da arte sobre a área CTS e a representatividade dos 4 periódicos nacionais referidos acima, procedemos com o segundo objetivo deste trabalho, isto é, fazer uma análise da produção CTS no último quinquênio (2009-2013) nos periódicos Ciência \& Educação, Ensaio, RBPEC e IENCI, comparando com os dados encontrados nas 13 pesquisas de estado da arte sintetizadas anteriormente.

\section{Procedimentos Metodológicos}

Para realização do segundo objetivo desta pesquisa nos direcionamos para a produção acadêmica publicada entre os anos 2009 e 2013, nos quatro periódicos nacionais mais investigados nas pesquisas em estado da arte anteriormente descritas: a revista Ensaio: Pesquisa em Educação em Ciências (Ensaio), a Revista Brasileira de 
Pesquisa em Educação em Ciências (RBPEC), a revista Investigações em Ensino de Ciências (IENCI) e a revista Ciência \& Educação (C\&E).

Com o intuito de comparar tendências nos trabalhos inventariados nas revisões bibliográficas com a produção recente em CTS, selecionamos os artigos publicados nos volumes dos anos de 2009 a 2013 das quatro revistas. A consulta foi realizada diretamente nos sites das revistas, buscando-se a palavra-chave 'CTS' nos títulos, nos resumos e/ou nas palavras-chave. Para refinar ainda mais a busca foi realizada a leitura dos resumos de cada trabalho. A partir desses critérios, foram encontrados 23 artigos nos quatro periódicos que abordavam o tema CTS na pesquisa, porém 2 trabalhos não tratavam diretamente do tema CTS e foram excluídos da análise, assim, o corpus de análise consiste em 21 artigos.

Após essa etapa, procedemos com a leitura atenta do conteúdo na íntegra de cada um dos artigos para uma análise mais criteriosa. Os artigos foram analisados de acordo com a Análise de Conteúdo descrito por Bardin (1977) em três fases: (i) préanálise, com a organização do material que constitui o corpus da investigação; (ii) descrição analítica, na qual são realizados procedimentos que classificam, categorizam e codificam o corpus da pesquisa de forma mais aprofundada se baseando nos referenciais teóricos; e (iii) interpretação referencial, nesta etapa ocorre a reflexão e a construção de significados embasadas nos materiais empíricos com mais intensidade (BARDIN,1977; TRIVIÑOS, 2012).

Tomamos como principal referência os seguintes descritores: distribuição por periódico, modalidades de formação investigada, foco temático de cada artigo, metodologias e técnicas utilizadas na coleta de dados e os referenciais teóricos que apoiaram os estudos.

\section{Análise comparativa entre as pesquisas de estado da arte e as publicações nos periódicos nacionais}

Quanto à distribuição por periódico no quinquênio analisado, a maior produção com a temática CTS foi nas revistas IENCI e C\&E com 7 artigos cada, seguida pelas revistas Ensaio onde foram encontrados 4 artigos e 3 artigos na RBPEC. Verificamos que a produção de trabalhos com a temática CTS se mostrou equilibrada no quinquênio, com exceção do ano de 2011 em que houve apenas uma pesquisa com a temática. A tabela 1 evidencia a distribuição anual dos artigos em cada um dos periódicos analisados. 
Tabela 1. Distribuição dos artigos sobre a tendência CTS no período de 2009-2013.

\begin{tabular}{l|c|c|c|c|c|c|c}
\hline \multirow{2}{*}{ Periódico } & \multicolumn{9}{|c|}{ Ano } & \multicolumn{2}{c}{ Total } \\
\cline { 2 - 8 } & $\mathbf{2 0 0 9}$ & $\mathbf{2 0 1 0}$ & $\mathbf{2 0 1 1}$ & $\mathbf{2 0 1 2}$ & $\mathbf{2 0 1 3}$ & $\mathbf{N}^{\mathbf{0}}$ & $\mathbf{\%}$ \\
\hline Ensaio & - & - & - & 3 & 1 & 4 & $19,1 \%$ \\
RBPEC & 1 & 2 & - & - & & 3 & $14,3 \%$ \\
IENCI & 4 & 1 & - & - & 2 & 7 & $33,3 \%$ \\
C\&E & 1 & 2 & 1 & 1 & 2 & 7 & $33,3 \%$ \\
Total & $\mathbf{6}$ & $\mathbf{5}$ & $\mathbf{1}$ & $\mathbf{4}$ & $\mathbf{5}$ & $\mathbf{2 1}$ & $\mathbf{1 0 0 \%}$ \\
\hline
\end{tabular}

Consideramos que este quinquênio (2009 a 2013) representa um verdadeiro "boom" de pesquisa na área CTS, com média de publicação de 4,2 artigos por ano. O crescimento de trabalhos foi exorbitante neste período ao compararmos com a revisão feita por Abreu et al. (2009), que ao analisarem todas as publicações de 10 periódicos nacionais até 2008 encontraram no conjunto de todas as publicações apenas 23 trabalhos com enforque CTS. Na época os autores enfatizaram que a produção acadêmica em CTS ainda era pouco expressiva $(0,78 \%)$ em relação a produção total do campo de pesquisa em ensino de ciências.

Em outro levantamento, quando Abreu et al. (2013) abrangeram a revisão para as atas de encontros nacionais perceberam que, proporcionalmente, os trabalhos em CTS estão mais evidentes nos encontros de pesquisa do que nos periódicos. Assim, o "boom" de trabalhos com abordagem CTS pode ser ainda mais significativo se forem considerados os trabalhos apresentados em eventos da área de educação em ciências. A exemplo disso, podemos citar as revisões de Fagundes et al. (2009), que identificaram um aumento de 08 para 108 trabalhos que abordaram a perspectiva CTS, do I (1997) para o VI (2007) ENPEC; e de Pansera-de-Araujo et al. (2009) que consideraram a distribuição dos trabalhos nos eventos aumentando consideravelmente ao longo dos anos ao mapearam trabalhos publicados em 4 eventos da área de ensino de Ciências, no período de 2003 a 2006, dos quais 110 (3,14\%) abordavam o enfoque CTS.

Essa maior concentração de pesquisas em atas de eventos em relação às revistas pode estar associada à maior periodicidade de edições de eventos que reúnem as comunidades científicas, ainda mais levando em consideração a interdisciplinaridade da área de Educação em Ciências, em que ocorrem eventos por áreas específicas da Física, Química e Biologia e também eventos interdisciplinares como o ENPEC. Além disso, o rigor requerido para publicação em periódicos, a demora dos pareceres de avaliação e o número reduzido de revistas especializadas dificultam a publicação das pesquisas.

Em relação às modalidades de formação e sujeitos investigados no quinquênio analisado, verificamos que a Educação Básica, em sua maioria alunos e professores do 
Ensino Médio, foi a modalidade mais pesquisada com $48 \%(\mathrm{n}=10)$ dos trabalhos (AMARAL et al., 2009; MARCONDES et al., 2009; SANTOS; MORTIMER, 2009; CARLETTO; PINHEIRO, 2010; KIST; FERRAZ, 2010; FIRME; AMARAL, 2011; BARBOSA et al., 2012; MUNDIN; SANTOS, 2012; FABRI; SILVEIRA, 2013; MENDES; SANTOS, 2013); seguida pela Educação Superior com 14\% das pesquisas $(n=3)$ (SILVA; CARVALHO, 2009; MACHADO; PINHEIRO, 2010; VERASZTO et al., 2013) e pela Educação Profissional ou Tecnológica com 14\% (n=3) (SILVEIRA; BAZZO, 2009; ARAÚJO; SILVA, 2012; SANTOS et al., 2012), nas quais os sujeitos eram alunos do curso de graduação; $5 \%(n=1)$ das pesquisas se aplica a todas as modalidades (BARBOSA; BAZZO, 2013) e 19\% $(n=4)$ não se enquadra especificamente em nenhuma modalidade de formação (PANSERA-DE-ARAUJO et al., 2009; SIQUEIRA-BATISTA et al., 2010; TEIXEIRA, 2010; CHRISPINO et al., 2013).

Resultados semelhantes foram encontrados por Miranda (2013b) nas teses e dissertações brasileiras, nas quais em relação ao nível de ensino $49 \%$ foram direcionadas à Educação Básica (31\% ao Ensino Médio e 18\% ao Ensino Fundamental), 14\%, ao Ensino Superior e 7\% ao Ensino Técnico. É interessante notar que este compasso demonstra que as pesquisas contidas nos periódicos têm refletido a produção de teses e dissertações defendidas ou em andamento de programas de pós-graduação e grupos de pesquisa da área da Educação em Ciências.

Nos levantamentos realizados por Sutil et al. (2008), Hunsche et al. (2009), Fagundes et al. (2009), Abreu et al. (2009; 2013), e Lima e Martins (2013), o conjunto de trabalhos revisados, tanto nos periódicos quanto em atas de eventos, também apontaram os professores como os principais sujeitos das investigações que abordavam concepções e práticas sobre CTS.

Outro aspecto a ser destacado é que a maioria das pesquisas foi desenvolvida no contexto do Ensino Médio, resultado também verificado por Fagundes et al. (2009) nas atas do ENPEC. Miranda (2013b) ao encontrar o mesmo resultado nas teses e dissertações brasileiras, discute que essa predominância de pesquisas no Ensino Médio pode estar relacionada ao fato de que a maioria das referências que discute as relações CTS estão nas áreas de Física, Química e Biologia, que são as áreas disciplinares pertencentes ao Ensino Médio.

Diante disso, percebemos que há uma grande lacuna de investigação a ser explorada pela comunidade de pesquisadores em relação à abordagem CTS em outros 
níveis de ensino, como a Educação Infantil, uma lacuna inclusive já apontada por Ferst (2013), a Educação de Jovens e Adultos e a Formação Continuada de professores.

Quanto ao foco temático de cada pesquisa, os trabalhos foram classificados em 4 eixos temáticos, a partir do objeto principal de estudo, quais são:

a) Implementação da abordagem CTS: reúne trabalhos que investigaram diretamente propostas metodológicas ou estratégias de ensino aplicadas em sala de aula com a abordagem CTS.

b) Concepções sobre CTS: agrupa trabalhos que tinham como objeto de estudo concepções, representações e percepções dos sujeitos sobre a abordagem CTS.

c) Livro didático: relaciona trabalhos que investigaram a abordagem CTS nos livros didáticos.

d) Revisão de literatura/teóricos: agrupa trabalhos que tiveram como foco específico estudos teóricos ou de revisão bibliográfica do tipo estado da arte.

Observamos, conforme Tabela 2, que no quinquênio houve predominância (52\%) de pesquisas que buscaram investigar como tema a implementação da abordagem CTS na sala de aula (MARCONDES et al., 2009; SANTOS; MORTIMER, 2009; CARLETTO; PINHEIRO, 2010; MACHADO; PINHEIRO, 2010; FIRME; AMARAL, 2011; BARBOSA et al., 2012; MUNDIN; SANTOS, 2012; SANTOS et al., 2012; FABRI; SILVEIRA, 2013; MENDES; SANTOS, 2013), seguida pela temática revisão de literatura/teóricos (24\%) (PANSERA-DE-ARAUJO et al., 2009; SIQUEIRABATISTA et al., 2010; TEIXEIRA, 2010; ARAÚJO; SILVA, 2012; BARBOSA; BAZZO, 2013; CHRISPINHO et al., 2013), concepções sobre CTS (19\%) (SILVA; CARVALHO, 2009; SILVEIRA; BAZZO, 2009; KIST; FERRAZ, 2010; VERASZTO et al., 2013), e uma pesquisa (AMARAL et al., 2009) que abordava como foco o livro didático. Os quatro periódicos analisados abordaram pelo menos dois dos três focos temáticos mais representativos, sendo que na revista $\mathrm{C} \& \mathrm{E}$ ocorreram os três focos.

Tabela 2. Foco temático das pesquisas sobre CTS por periódico (2009-2013).

\begin{tabular}{lcccccc}
\hline \multicolumn{1}{c}{ Foco temático } & C\&E & Ensaio & RBPEC & IENCI & Total & \% \\
\hline Implementação da abordagem & 3 & 2 & - & 5 & 10 & $52 \%$ \\
CTS & & & & & & \\
Revisão de literatura/teóricos & 2 & 2 & 2 & - & 6 & $24 \%$ \\
Concepções sobre CTS & 2 & - & 1 & 1 & 4 & $19 \%$ \\
Livro didático & & & & 1 & 1 & $5 \%$ \\
\hline Total & & & & 21 & $100 \%$ \\
\hline
\end{tabular}


Verificamos que o foco temático "implementação da abordagem CTS" em sala de aula vem recebendo maior atenção por parte dos pesquisadores nos últimos anos, o que é interessante, pois responde a uma preocupação apresentada por pesquisadores da área quanto à efetivação da proposta CTS no contexto escolar. Por exemplo, Cachapuz et al. (2008) e Hunsche et al. (2009) identificaram em periódicos internacionais (1993 a 2002) e nacionais (1998-2008), respectivamente, o predomínio de pesquisas que discutiam os pressupostos teóricos do campo CTS e poucas pesquisas refletindo sobre efetivas implementações no ensino de ciências, revelando a carência de estudos sobre a intervenção da abordagem CTS no contexto escolar. No estudo de Pansera-de-Araujo et al. (2009) as investigações sobre a intervenção de práticas educativas CTS são apontadas como incipientes nas atas dos diferentes eventos analisados (2003-2006) e os autores apontam com inquietação para a necessidade de se investigar outras bases de dados para verificar se essa tendência se confirma. Além disso, Sutil et al. (2008) apontam que nas pesquisas envolvendo intervenções, geralmente os autores buscaram indícios de sucesso ou fracasso das estratégias de ensino e aprendizagem e de propostas; a avaliação, como componente de um processo didático, foi praticamente inexistente nos trabalhos.

Um ponto interessante de se observar na análise das pesquisas em estado da arte é que, se por uma lado o foco temático implementações CTS no contexto escolar aparece com menor ênfase nos periódicos internacionais e nacionais (até 2008, pois tanto nosso estudo quanto de Lima e Martins (2013) destacam a temática implementações nos últimos anos) por outro, tanto nas teses e dissertações esta temática aparece como predominante conforme a revisão de Miranda (2013a), quanto nas atas de encontros da área de educação em ciências, segundo Fagundes et al. (2009) e Abreu et al. (2013). Estes últimos autores justificam que a maior ocorrência da temática implementações CTS no contexto escolar nos eventos possivelmente está relacionada com a própria natureza desses encontros.

Para Hunsche et al. (2009), a pouca ênfase apontada pelas revisões bibliográficas de pesquisas sobre implementação da abordagem CTS até aquele momento, poderia sinalizar prudência por parte dos pesquisadores, isto é, certa precaução para evitar transposições acríticas para a prática de sala de aula dos pressupostos CTS.

No entanto, o despontamento de pesquisas sobre intervenções no contexto escolar a partir de 2008 e sua forte presença em teses e dissertações e atas de eventos, constitui um elemento bastante positivo, pois de acordo com Sutil et al. (2008) a 
inserção de pressupostos do movimento CTS no ensino de Ciências precisa ser estruturado na sala de aula e também na formação de professores.

O segundo foco temático mais representativo "revisão de literatura/teóricos" com 24\% (n=6), teve dois trabalhos de revisão bibliográfica (PANSERA-DE-ARAUJO et al., 2009; CHRISPINHO et al., 2013), e quatro de ensaios teóricos abordando assuntos como nanotecnologia (SIQUEIRA-BATISTA et al., 2010), análise teóricoconceitual do discurso CTS para o ensino de ciências (TEIXEIRA, 2010), discussão da possibilidade de integrar os pressupostos CTS e Trabalho e Educação ao currículo da Educação Profissional e Tecnológica (ARAÚJO; SILVA, 2012) e o uso de documentários como metodologia para o ensino CTS (BARBOSA; BAZZO, 2013). Os trabalhos teóricos demonstram a preocupação dos pesquisadores brasileiros com a fundamentação do aporte teórico-metodológica da área CTS.

Apesar da importância dessa temática, os trabalhos de Fagundes et al. (2009), Abreu et al. (2009), na época sinalizaram que esse foco temático apresentava pouca representatividade, nas atas das seis primeiras edições do ENPEC e em periódicos nacionais, respectivamente. Lima e Martins (2013) apontam que nos periódicos internacionais o número de trabalhos teóricos é maior comparado com a produção brasileira.

Em relação aos trabalhos com foco temático "concepções sobre CTS", estes foram desenvolvidos com professores (KIST; FERRAZ, 2010), graduandos (SILVA; CARVALHO, 2009; VERASZTO et al., 2013) e com empreendedores e gestores de empresas tecnológicas (SILVEIRA; BAZZO, 2009). Verificamos que ainda há grande preocupação dos pesquisadores com as concepções das relações CTS de professores e futuros professores, o que também foi constatado por Fagundes et al. (2009) nas atas das seis primeiras edições do ENPEC e Lopes et al. (2009) nas edições de 2000 a 2007 do mesmo evento; o mesmo resultado também foi encontrado por Gonçalves e Menezes (2013), Hunsche et al. (2009) e Ferst (2013) em periódicos nacionais.

Fagundes et al. (2009) ressaltam que nos trabalhos analisados é enfatizado que as concepções sobre CTS e os modos de ensinar dos professores continuam iguais mesmo depois de cerca de 40 anos do início do movimento CTS. De acordo com Gonçalves e Menezes (2013), as arraigadas concepções de ciência e tecnologia como salvacionista e linear, incompatíveis com a proposta didática sob a perspectiva CTS, trazem certa preocupação para os pesquisadores, pois para a implementação da 
abordagem CTS no ensino de ciências é fundamental a compreensão da natureza social da ciência e da tecnologia.

No que se refere às metodologias e técnicas, verificamos que nas pesquisas são utilizadas duas ou mais técnicas combinadas para construção de dados (38\%), seguida por estudo teórico $(28,5 \%)$ e entrevistas/questionários (14\%) (Tabela 3). Observamos que esse descritor "metodologia e técnicas" não foi analisado em nenhuma das pesquisas sobre estado da arte consideradas.

Tabela 3. Metodologias e técnicas de coleta de dados das pesquisas.

\begin{tabular}{|c|c|c|c|c|c|c|}
\hline \multirow{2}{*}{ Metodologias } & \multirow{2}{*}{ Ensaio } & \multirow{2}{*}{ RBPEC } & \multirow{2}{*}{ IENCI } & \multirow{2}{*}{ C\&E } & \multicolumn{2}{|c|}{ Total } \\
\hline & & & & & $\mathbf{N}$ & $\%$ \\
\hline $\begin{array}{l}\text { Duas ou mais técnicas } \\
\text { combinadas }\end{array}$ & 2 & - & 5 & 1 & 8 & $38 \%$ \\
\hline $\begin{array}{lll}\begin{array}{l}\text { Estudo } \\
\text { literatura }\end{array} & \text { teórico/revisão } & \text { de } \\
\end{array}$ & 1 & 2 & 1 & 2 & 6 & $28,5 \%$ \\
\hline $\begin{array}{l}\text { Entrevistas escritos e orais e } \\
\text { questionários }\end{array}$ & - & 1 & - & 2 & 3 & $14 \%$ \\
\hline Não especificado & 1 & - & 1 & - & 2 & $9,5 \%$ \\
\hline Grupo focal & - & - & - & 1 & 1 & $5 \%$ \\
\hline Observação & - & - & - & 1 & 1 & $5 \%$ \\
\hline Total & 4 & 3 & 7 & 7 & 21 & $100 \%$ \\
\hline
\end{tabular}

Observamos que as metodologias de pesquisa acompanham os focos temáticos mais representativos (implementações, revisões teóricas e concepções), o que representa coerência teórico-metodológica, isto é, os métodos de investigação adotados nas pesquisas são coerentes com os objetivos das mesmas.

Por exemplo, a maior parte dos trabalhos que buscam analisar implementações da abordagem CTS na sala de aula, lançam mão de diferentes técnicas de coleta como: observação, diário de campo, gravações em áudio e vídeo, análise de materiais construídos, questionários, entrevistas, grupo focal. Segundo André et al. (2010) a combinação de diferentes formas de coleta de dados representa um avanço nas pesquisas, pois tal variedade de fontes de coleta indica uma abordagem mais ampla das questões, o que traz maior riqueza para as pesquisas.

De acordo com Andrade (2007), a utilização de mais de um instrumento de coleta de dados, em geral, a entrevista combinada com alguma outra, revela a preocupação dos pesquisadores em olhar o objeto investigado sob diferentes perspectivas o que parece adequado em se tratando da área de educação em que as questões são, em geral, muito complexas. As entrevistas e questionários são, segundo 
André (2009), coerentes com a temática concepções, isto por que a intenção dos pesquisadores nesse tipo de trabalhos é de conhecer as concepções, representações, percepções e opiniões dos sujeitos.

Quanto aos referenciais teóricos que embasaram os estudos sobre CTS nos 21 artigos analisados no período de 2009 a 2013, verificamos que há trabalhos que se constituíram como referência para a área uma vez que compareceram com maior frequência nas pesquisas. Identificamos e listamos abaixo as 12 referências mais citadas nas pesquisas. O número no final da referência indica em quantos trabalhos ela compareceu dentre os 21 analisados:

1. SANTOS, W. L. P.; MORTIMER, E. F. Uma análise de pressupostos teóricos da abordagem C-T-S (Ciência-Tecnologia-Sociedade) no contexto da educação brasileira. Ensaio: Pesquisa em Educação em Ciências, v. 2, n. 2, p. 133-162, 2002. (9).

2. AULER, D. Alfabetização científico-tecnológica: Um novo 'paradigma'? Ensaio: Pesquisa e Educação em Ciência, v. 5, n. 1, p. 1-16. 2003. (5).

3. AULER, D.; DELIZOICOV, D. Alfabetização científico-tecnológica para quê? Ensaio: Pesquisa em Educação em Ciências, v. 3, n. 2, p. 105-116, 2001. (5).

4. SANTOS, W. L. P.; SCHNETZLER, R. P. Ciência e educação para a cidadania. In: CHASSOT, A.; OLIVEIRA, R. J. (Org.). Ciência, ética e cultura na educação. São Leopoldo: Editora Unisinos, 1997. (5).

5. BAZZO, W. A. Ciência, tecnologia e sociedade: o contexto da educação tecnológica. Florianópolis: Ed. da UFSC, 1998 (4).

6. PINHEIRO, N. A. M.; SILVEIRA, R. M. C. F; BAZZO, W. A. Ciência, tecnologia e sociedade: a relevância do enfoque CTS para o contexto do ensino médio. Ciência \& Educação, v. 13, v. 1, p. 71-84, 2007. (4).

7. SANTOS, W. L. P. dos. Educação científica humanística em uma perspectiva freireana: resgatando a função do ensino de CTS. Alexandria: Revista de Educação em Ciência e Tecnologia, v. 1, n. 1, p. 109-131, 2008. (4).

8. AULER, D. Interações entre ciência-tecnologia-sociedade no contexto da formação de professores de ciências. 2002. 248 f. Tese (Doutorado em Educação) Universidade Federal de Santa Catarina, Florianópolis, 2002. (3).

9. AULER, D.; BAZZO, W. A. Reflexões para implementação do movimento CTS no contexto educacional brasileiro. Ciência \& Educação, v. 7, n. 1, p. 1-13, 2001. (3). 
10. AULER, D.; DELIZOICOV, D. Ciência-tecnologia-sociedade: relações estabelecidas por professores de ciências. Revista Electrónica de Enseñanza de las Ciencias, v. 5, n. 2, p. 337-355, 2006. (3).

11. BAZZO, W. A; VON LINSINGEN, I.; PEREIRA, L. T. V. Introdução aos estudos CTS (Ciência, Tecnologia e Sociedade). Madri: Organização dos Estados Iberoamericanos, 2003. (3).

12. CHASSOT, A. Alfabetização científica: questões e desafios para a educação. Ijuí: Unijuí, 2000. (3).

Dentre estes referenciais, os autores que mais se destacaram por terem vários de seus trabalhos citados, como único autor ou em colaboração, e que parecem ser os principais expoentes sobre o assunto CTS nas pesquisas brasileiras são: Wildson Luiz Pereira dos Santos, Décio Auler, Walter Antônio Bazzo, Demétrio Delizoicov e Eduardo Fleury Mortimer. Entre as referências internacionais os autores que se destacam são: Aikenhead (1994, 2006), Acevedo (1997, 2001), Acevedo, Vasquez e Manassero (2003), Garcia, Cerezo e López (1996).

Esses mesmos autores nacionais e internacionais foram os mais recorrentes também no levantamento realizado por Abreu et al. (2009) e Lima e Martins (2013), os quais são considerados um referencial importante para os estudos da abordagem CTS.

Em interessante levantamento desenvolvido por Chrispino et al. (2013) com objetivo de mapear os autores e trabalhos mais citados nas pesquisas brasileiras, eles chegaram ao conjunto de 13 trabalhos (um total de 15 autores, dez brasileiros e cinco estrangeiros), que são: Santos e Mortimer (2002), Garcia, Cerezo e López (1996), Auler e Bazzo (2001), Auler (2002), Bazzo (1998), Bazzo et al. (2003), Auler e Delizoicov (2006), Acevedo (1996), Amorim (1997), Cruz e Zylbersztajn (2001), Solomon (1993), Acevedo (1995) e Auler e Delizoicov (2001). Em nossa pesquisa encontramos 8 dos trabalhos mais citados dos 13 autores que representam, segundo Chrispino et al. (2013), o conhecimento acumulado na área de CTS no ensino de ciências. Por isso essas referências podem ser encaradas como leitura obrigatória num curso para entendimento do que seja e o que produz a área CTS.

Os autores brasileiros mais citados como Décio Auler, Walter Bazzo, Wildson Santos, Eduardo Mortimer e Demetrio Delizoicov, se estabelecem como referência para a comunidade e são considerados como pertencentes à classe dos fundadores do que Dagino (2008 apud ABREU et al., 2009) chama de Pensamento Latino Americano de Ciência, Tecnologia e Sociedade, em pleno processo de desenvolvimento no Brasil. 
Segundo Abreu et al. (2013), o pensamento latino americano em CTS se diferencia das linhas norte-americanas e europeias devido às condições históricas de colonização econômica e cultural desses países, que produziram ao longo do tempo desigualdades sociais e condição de periferia econômica da ordem mundial. Esse contexto desenvolveu na América Latina um pensamento crítico sobre CTS, que envolve a melhoria de qualidade de vida, consolidação de uma sociedade democrática e a construção de práticas de cidadania e responsabilidade social. Ainda de acordo com Abreu et al. (2013) existe dentro do pensamento brasileiro em CTS uma preocupação em negar mitos salvacionistas de ciência e tecnologia, defendendo uma educação científica voltada para uma leitura mais crítica das relações CTS.

É importante ressaltar o relevo que os pressupostos freireanos recebem nas pesquisas nacionais, apontados pelos trabalhos de revisão, sendo por isso, destacados como uma tendência no contexto brasileiro (SUTIL et al., 2008; AULER et al., 2009; HUNSCHE et al., 2009; GONÇALVES; MENEZES, 2013; ABREU et al., 2013; LIMA; MARTINS, 2013).

A recorrente articulação do pensamento de Paulo Freire e a perspectiva CTS, ocorre devido os dois pressupostos, e especialmente a educação emancipatória de Paulo Freire, visarem uma formação humanística para a cidadania com princípios e práticas pautadas na promoção de uma educação em ciências comprometida com a transformação social, a emancipação dos sujeitos e a justiça social (ABREU et al., 2013; LIMA; MARTINS, 2013).

Apesar da aparente semelhança e tentativa de articulação entre a abordagem CTS e os pressupostos freireanos, o estudo de revisão feito por Auler et al. (2009) evidenciou que há dissonância entre as duas abordagens nas pesquisas CTS que utilizam temas. Pois em Freire, o tema surge com uma efetiva participação da comunidade escolar e são constituídos de manifestações locais de contradições presentes na dinâmica social, além disso, as disciplinas envolvidas não ficam restritas a uma área de conhecimento. Nos encaminhamentos dados nas pesquisas que utilizam temas com enfoque CTS, de forma diferente dos pressupostos freireanos, a escolha dos temas é hegemonicamente definida pelo professor, os temas são de abrangência mais geral, não vinculados a contextos específicos e as disciplinas envolvidas ficam restritas à área das ciências, às disciplinas de Biologia, Física, Química, Geologia e Matemática.

Ainda com relação aos referenciais, Chrispino et al. (2013) chamam atenção em sua revisão para duas ausências nos fundamentos teóricos das pesquisas, em primeiro 
lugar que em geral os autores tidos como as fontes primárias, isto é, os fundadores da área CTS, não são citados nas investigações brasileiras. Os autores justificam essa ausência devido: a qualidade de síntese dos trabalhos nacionais dos fundamentos da área de CTS, a barreira do idioma e a inexistência de exemplares disponíveis das fontes primárias em meio digital. Em segundo, Chrispino et al. (2013) discutem a necessidade do campo CTS se desenvolver a partir de uma visão interdisciplinar e contextualizada, superando a possível restrição de produção da área de ensino, para as áreas fundantes de CTS (Filosofia, Sociologia, História, Cultura, Economia, Política, Meio Ambiente), que permitiriam estruturar materiais e aprimorar análises e processos de formação mais críticos.

\section{Considerações finais}

O campo de estudos e pesquisa com abordagem CTS tem expandido e se disseminado consideravelmente nas últimas décadas, o que implica em periodicamente revisar tal produção acadêmica. Identificar seus principais resultados, tendências e possíveis contribuições para a melhoria do ensino de ciências e o desenvolvimento de novas linhas de investigação.

A partir da sumarização feita de pesquisas de revisão bibliográfica e da análise comparativa com pesquisas com enfoque CTS do último quinquênio (2009-2013), verificamos que a linha de pesquisa CTS continua em processo de expansão na área de pesquisa do ensino de ciências. Tanto nos periódicos nacionais quanto nos eventos ocorre a disseminação, no entanto ela é mais frequente nos eventos acadêmicos da área. que nas publicações em periódicos.

Com base no estudo realizado é possível afirmar que algumas tendências de pesquisa em CTS continuam as mesmas, como as modalidades de formação e sujeitos mais investigados, isto é, Educação Básica (mais especificamente o Ensino Médio) e professores, respectivamente. Permanece também interesse dos pesquisadores em reflexões teóricas, seja através da fundamentação teórica da área CTS baseada em autores nacionais - na busca por uma identidade própria para o pensamento CTS brasileiro, em especial nos pressupostos freireanos - seja na sistematização frequente da produção na área.

Uma tendência que se alterou ao longo da última década foi o foco das pesquisas, que recentemente tem investido mais em investigar intervenções em situações de ensino CTS em sala de aula e menos em pesquisas sobre concepções de 
alunos e professores sobre CTS, que foram a tônica das produções até o início desta década.

Consideramos que o presente estudo, através da síntese de trabalhos de revisão bibliográfica juntamente com a análise comparativa de periódicos, possibilitou uma visualização do atual estado do conhecimento no campo CTS no cenário brasileiro na área da educação em ciências identificando tendências de pesquisa, situando referências e autores considerados importantes expoentes da área, apontando lacunas de pesquisa. Os resultados desta pesquisa constituem-se em uma contribuição para a reflexão, o debate, a consolidação e o avanço da pesquisa na área CTS no contexto brasileiro.

\section{Referências}

ABREU, T. B.; FERNANDES, J. P; MARTINS, I. Uma análise qualitativa e quantitativa da produção cientifica sobre CTS (ciência, tecnologia e sociedade) em periódicos da área de ensino de ciências no Brasil. In: ENCONTRO NACIONAL DE PESQUISA EM EDUCAÇÃO EM CIÊNCIAS, 7, 2009. Florianópolis. Anais... Florianópolis, 2009.

ABREU, T. B; FERNANDES, J. P; MARTINS, I. Levantamento sobre a produção CTS no Brasil no período de 1980-2008 no campo de ensino de ciências. Alexandria Revista de Educação em Ciência e Tecnologia, v.6, n.2, p. 3-32, jun., 2013.

AMARAL, C. L. C.; XAVIER, E. S.; MACIEL, M. L. Abordagem das relações ciência/tecnologia/sociedade nos conteúdos de funções orgânicas em livros didáticos de química do ensino médio. Investigações em Ensino de Ciência, v. 14, n. 1, p. 101-114, 2009.

ANDRADE, R. R. M. Pesquisas sobre formação de professores: uma comparação entre os anos 90 e 2000. In: REUNIÃO ANUAL DA ANPED, 1, 2007, Caxambu. Anais... Caxambu, 2007.

ANDRÉ, M. A produção acadêmica sobre formação de professores: um estudo comparativo das dissertações e teses defendidas nos anos 1990 e 2000. Form. Doc., v. 1, n. 1, p. 41-56, ago/dez. 2009.

ANDRÉ, M. Formação de professores: a constituição de um campo de estudos. Educação, v. 33, n. 3, p. 174-181. 2010.

ANDRÉ, M; SIMÕES, R. H. S; CARVALHO, J. M; BRZEZINSKI, I. Estado da Arte da Formação de Professores no Brasil. Educação \& Sociedade, v. 68, p. 301-309. 1999.

ARAÚJO, A. B.; SILVA, M. A. Ciência, tecnologia e sociedade; trabalho e educação: possibilidades de integração no currículo da educação profissional tecnológica. Rev. Ensaio, v. 14, n. 01, p. 99-112, 2012. 
AULER, D; BAZZO, W. A. Reflexões para a implementação do movimento CTS no contexto educacional brasileiro. Ciência \& Educação, v.7, n.1, p.1-13, 2001.

AULER, D; DALMOLIN, A. M. T; FENALTI, V. S. Abordagem Temática: natureza dos temas em Freire e no enfoque CTS. Alexandria Revista de Educação em Ciência e Tecnologia, v.2, n.1, p.67-84. 2009.

BARBOSA, L. C. A.; BAZZO, W. A. O uso de documentários para o debate ciênciatecnologia-sociedade (CTS) em sala de aula. Rev. Ensaio, v.15, n. 3, p. 149-161, 2013.

BARBOSA, L. G. D’C.; LIMA, M. E. C. C.; MACHADO, A. H. Controvérsias sobre o aquecimento global: circulação de vozes e de sentidos produzidos em sala de aula. Rev. Ensaio, v. 14, n. 1, p.113-130, 2012.

BARDIN, L. Análise de conteúdo. Lisboa: Edições 70. 1995.

CACHAPUZ, A; PAIXÃO, F; BERNARDINO LOPES, J; GUERRA, C. Do Estado da Arte da Pesquisa em Educação em Ciências: Linhas de Pesquisa e o Caso "CiênciaTecnologia-Sociedade". Alexandria Revista de Educação em Ciência e Tecnologia, v.1, n.1, p. 27-49, 2008.

CARLETTO, M. R.; PINHEIRO, N. A. M. Subsídios para uma prática pedagógica transformadora: contribuições do enfoque CTS. Investigações em Ensino de Ciências, v. 15 , n. 3, p. $507-525,2010$.

CHRISPINO, A; LIMA, L. S; ALBUQUERQUE, M. B; FREITAS, A. C. C; SILVA, M. A. F. B. A área CTS no Brasil vista como rede social: onde aprendemos? Ciência \& Educação, v. 19, n. 2, p. 455-479, 2013.

FABRI, F.; SILVEIRA, R. M. C. F. O ensino de ciências nos anos iniciais do ensino fundamental sob a ótica CTS: uma proposta de trabalho diante dos artefatos tecnológicos que norteiam o cotidiano dos alunos. Investigações em Ensino de Ciências, v. 18, n. 1, p. $77-105,2013$.

FAGUNDES, S. M. K; PICCINI, I. P; IAMARQUE, T; TERRAZZAN, E. A. Produções em educação em ciências sob a perspectiva CTS/CTSA. In: ENCONTRO NACIONAL DE PESQUISA EM EDUCAÇÃO EM CIÊNCIAS, 7, 2009. Florianópolis. Anais... Florianópolis, 2009.

FERREIRA, N. S. A. As pesquisas denominadas "Estado da Arte". Educação \& Sociedade, v. 21, n. 79, p. 257-272. 2002.

FERST, E. M. A abordagem CTS no ensino de ciências naturais: possibilidades de inserção nos anos iniciais do ensino fundamental. Revista EDUCAmazônia - Educação Sociedade e Meio Ambiente, v. 11, n. 2, p. 276-299. 2013.

FIRME, R. N.; AMARAL, E. M. R. Analisando a implementação de uma abordagem CTS na sala de aula de química. Ciência \& Educação, v. 17, n. 2, p. 383-399, 2011. 
GONÇALVES, R. S; MENEZES, P. H. D. Educação em Ciências balizada pelo enfoque CTS: perspectivas e possibilidades para o ensino de ciências da escola básica. In: ENCONTRO NACIONAL DE PESQUISA EM EDUCAÇÃO EM CIÊNCIAS, 9, 2013. Águas de Lindóia. Anais... Águas de Lindóia, 2013.

HUNSCHE, S.; DALMOLIN, A. M. T.; ROSO, C. C.; SANTOS, R. A.; AULER, D. O enfoque CTS no contexto brasileiro: caracterização segundo periódicos da área de educação em ciências. In: ENCONTRO NACIONAL DE PESQUISA EM EDUCAÇÃO EM CIÊNCIAS, 7., 2009, Florianópolis. Anais... Florianópolis, 2009.

KIST, C. P.; FERRAZ, D. F. Compreensão de professores de biologia sobre as interações entre ciência, tecnologia e sociedade. Revista Brasileira de Pesquisa em Educação em Ciências, v. 10, n. 1, 2010.

LIMA, A; MARTINS, I. As interfaces entre a abordagem CTS e as questões sociocientíficas nas pesquisas em educação em ciências. In ENCONTRO NACIONAL DE PESQUISA EM EDUCAÇÃO EM CIÊNCIAS, 9, 2013. Águas de Lindóia. Anais... Águas de Lindóia, 2013.

LOPES, N. C; ANDRADE, J. A. N; QUEIRÓS, W. P; SOUZA, R. R; NARDI, R; CARVALHO, W. L. P. Tendências do movimento CTS em dois eventos nacionais da área de ensino de ciências. In: XVIII SIMPÓSIO NACIONAL DE ENSINO DE FÍSICA, 15, 2009, Vitória. Anais... Vitória, 2009.

MACHADO, V.; PINHEIRO, N. A. M. Investigando a metodologia dos problemas geradores de discussões: aplicações na disciplina de física no ensino de engenharia. Ciência \& Educação, v. 16, n. 3, p. 525-542, 2010.

MARCONDES, M. E. R.; CARMO, M. P.; SUART, R. C.; SILVA, E. L.; SOUZA, F. L.; SANTOS JR., J. B.; AKAHOSHI, L. H. Materiais instrucionais numa perspectiva CTSA: uma análise de unidades didáticas produzidas por professores de química em formação continuada. Investigações em Ensino de Ciências, v. 14, n. 2, p. 281-298, 2009.

MENDES, M. R. M.; SANTOS, W. L. P. Argumentação em discussões sociocientíficas. Investigações em Ensino de Ciências, v. 18, n. 3, p. 621-643, 2013.

MIRANDA, E. M. Análise das principais tendências da perspectiva ciência, tecnologia e sociedade (CTS) em teses e dissertações brasileiras das áreas de educação e ensino de ciências. Enseñanza de las Ciencias, v. 2013, p. 2214-2218, 2013 a.

MIRANDA, E. M. Panorama das teses e dissertações brasileiras e portuguesas sobre educação ciência, tecnologia e sociedade. Enseñanza de las Ciencias, v. 2013, p. 22192224, 2013b.

MUNDIN, J. V.; SANTOS, W. L. P. Ensino de ciências no ensino fundamental por meio de temas sociocientíficos: análise de uma prática pedagógica com vista à superação do ensino disciplinar. Ciência \& Educação, v. 18, n. 4, p. 787-802, 2012 
PANSERA-DE-ARAÚJO, M. C; GEHLEN, S. T; MEZALIRA, S. M; SCHEID, M. N. Enfoque CTS na pesquisa em educação em ciências: extensão e disseminação. Revista Brasileira de Pesquisa em Educação em Ciências, v. 9, n. 3, p. 1-21, 2009.

SANTOS, L. P; MORTIMER, E. F. Uma análise de pressupostos teóricos da abordagem C-T-S (Ciência-Tecnologia-Sociedade) no contexto da educação brasileira. Rev. Ensaio, v. 2, n. 2, p. 1-23, 2002.

SANTOS, M. S.; AMARAL, C. L. C.; MACIEL, M. D. Tema sociocientífico "cachaça" em aulas práticas de química na educação profissional: uma abordagem CTS. Rev. Ensaio, v. 14, n. 01, p. 227-239. 2012.

SANTOS, W. L. P.; MORTIMER, E. F. Abordagem de aspectos sociocientíficos em aulas de ciências: possibilidades e limitações. Investigações em Ensino de Ciências, v. 14, n. 2, p. 191-218, 2009.

SILVA, L. F.; CARVALHO, L. M. Professores de física em formação inicial: o ensino de física, a abordagem CTS e os temas controversos. Investigações em Ensino de Ciências, v. 14, n. 1, p. 135-148, 2009.

SILVEIRA, R. M. C. F.; BAZZO, W. Ciência, tecnologia e suas relações sociais: a percepção de geradores de tecnologia e suas implicações na educação tecnológica. Ciência \& Educação, v. 15, n. 3, p. 681-694, 2009.

SIQUEIRA-BATISTA, R.; SILVA, L, M,; SOUZA, R. R. M.; PIRES-DO-PRADO, H. J.; SILVA, C. A.; RÔÇAS, G.; OLIVEIRA, A. L.; HELAYËL-NETO, J. A. Nanociência e nanotecnologia como temáticas para discussão de ciência, tecnologia, sociedade e ambiente. Ciência \& Educação, v. 16, n. 2, p. 479-490, 2010.

STRIEDER, R. B; KAWAMURA, M. R. D. Discussões sobre CTS no âmbito da Educação em Ciências: parâmetros e enfoques. In: ENCONTRO NACIONAL DE PESQUISA EM EDUCAÇÃO EM CIÊNCIAS, 8, 2011, Campinas. Anais... Campinas, 2011.

SUTIL, N; BORTOLETTO, A; CARVALHO, W; CARVALHO, L. M. O. CTS e CTSA em periódicos nacionais em ensino de ciências/física (2000- 2007): considerações sobre a prática educacional em física. In: ENCONTRO DE PESQUISA EM ENSINO DE FÍSICA, 11, 2008, Curitiba. Anais... Curitiba. 2008.

TEIXEIRA, F. M. Discurso e Ensino das Ciências. Revista Brasileira de Pesquisa em Educação em Ciências, v. 10, n. 3, p. 87-100. 2010.

TRIVIÑOS, A. N. S. Introdução à pesquisa em Ciências Sociais: a pesquisa qualitativa em educação. São Paulo: Atlas. 2012.

VERASZTO, E. V.; SILVA, D.; CAMARGO, E. P.; FILHO, J. B. Concepções de tecnologia de graduandos do estado de São Paulo e suas implicações educacionais: breve análise a partir de modelagem de equações estruturais. Ciência \& Educação, v. 19, n. 3, p. 761-779, 2013. 
LILLIANE MIRANDA FREITAS. Graduada em Licenciatura Plena em Ciências Biológicas pela Universidade Federal do Pará. Mestre e doutoranda em Educação em Ciências pelo Programa de Pós-Graduação em Educação em Ciências e Matemáticas do Instituto de Educação Matemática e Científica - IEMCI/UFPA. Professora Assistente III na Faculdade de Ciências Naturais do Instituto de Estudos Costeiros - IECOS, Campus Universitário de Bragança - Universidade Federal do Pará.

EVANDRO GHEDIN. Possui graduação em Licenciatura em Filosofia pela Universidade Católica de Brasília (1995), graduação em Bacharelado em Filosofia pelo Centro de Estudos de Comportamento Humano (1992), especialização em Antropologia na Amazônia pela Universidade Federal do Amazonas (1997), especialização em Filosofia e Existência pela Universidade Católica de Brasília (1999), mestrado em Educação pela Universidade Federal do Amazonas (2000), doutorado em Educação pela Universidade de São Paulo (2004), pós-doutorado pela Universidade de São Paulo (2010). Atualmente é Professor Doutor da Universidade Estadual de Roraima e Professor Pesquisador da REAMEC - Rede Amazônica de Educação em Ciências e Matemática.

Recebido: 04 de setembro de 2014

Revisado: 30 de junho de 2015

Aceito: 18 de agosto de 2015 